\title{
Global Quality Standard for Identification and Management of Severe Asthma
}

\author{
John Haughney • Tonya A. Winders · Steve Holmes · Pascal Chanez • \\ Hannah Saul - Andrew Menzies-Gow on behalf of the PRECISION Improve Access to Better Care Task Force
}

Received: June 18, 2020 / Published online: July 28, 2020

(C) The Author(s) 2020

\section{ABSTRACT}

Introduction: Severe asthma is a debilitating, life-threatening disease associated with substantial global morbidity, mortality, and health care resource utilization. Patients may not receive guideline-directed medical care for severe asthma. Moreover, viable precision-based assessment tools and newer preventive therapies that can reduce the frequency of exacerbations and associated functional impact are underused. As a result, high rates of poorly

Digital Features To view digital features for this article go to https://doi.org/10.6084/m9.figshare.12656966.

J. Haughney $(\bowtie)$

Glasgow Clinical Research Facility, Queen Elizabeth

University Hospital, Glasgow, UK

e-mail: j.haughney@abdn.ac.uk

T. A. Winders

Allergy \& Asthma Network, Vienna, VA, USA

S. Holmes

Park Medical Practice, Shepton Mallet, UK

P. Chanez

Aix Marseille Université, Clinique des bronches, Allergie Et Sommeil/APHM, Marseille C2VN Center INSERM INRA UMR1062, Marseille, France

H. Saul

AstraZeneca, Cambridge, UK

A. Menzies-Gow

Royal Brompton and Harefield NHS Foundation

Trust, London, UK controlled severe asthma persist, and patient health-related quality of life suffers.

Methods: In 2019, the Improve Access to Better Care Task Force of the PRECISION Steering Committee set out to develop a global template on quality standards for severe asthma care to support improved access to and delivery of quality care. This Quality Standard is grounded in the vast body of published evidence available for severe asthma care, published clinical guidelines (i.e., from the Global Initiative for Asthma in 2019 and the European Respiratory Society/American Thoracic Society in 2014), and the 2018 PRECISION-supported Charter to Improve Patient Care in Severe Asthma.

Results: The Quality Standard developed emphasizes four key elements aimed at optimizing clinical care and outcomes in severe asthma: (1) organization of services, (2) timely identification and referral for suspected severe asthma, (3) specialized assessment and management of severe asthma to optimize outcomes, and (4) patient-centric care and shared decision-making that is reflective of the patient's expectations, priorities, and values. Four key Quality Statements are provided, along with quality metrics and strategies for local adaptation to optimize implementation.

Conclusion: This Global Quality Standard is intended to mobilize policymakers, health care providers, and patient advocacy groups to build consensus on the definition and expectations of quality care in severe asthma, to promote 
patient-centric care, to identify gaps in care and areas for improvement, and systematically implement improvement measures and outcomes and to reduce the burden of illness for patients with severe asthma.

\section{PLAIN LANGUAGE SUMMARY}

Although only $10 \%$ of patients with asthma have severe disease, these patients use up to half of all health care resources used to treat asthma. For the patient, severe asthma is associated with substantial morbidity, increased risk of death, and poor quality of life. Effective treatments for severe asthma are available, yet access to these treatments varies for many patients around the globe, and they are not always used effectively when available. A task force of leading global asthma experts was recently assembled to develop global standards to support improved access and delivery of quality care for patients with severe asthma. The task force identified four key elements to optimize management and outcomes: (1) coordination of services, (2) timely detection and referral of patients with severe asthma, (3) use of guideline-recommended assessments and therapies, and (4) integration of patient expectations and values when making treatment decisions. This Quality Standard details each of these elements by providing supporting rationale, ways to measure improvements in each area, and strategies to implement these elements at local clinics around the world. Ultimately, this Quality Standard is intended to help policymakers, health care providers, patient advocacy groups, and other key stakeholders build consensus on the requirements for quality care in severe asthma to improve patient care while also reducing the overall global burden of severe asthma.

Keywords: Guideline-directed medical therapy; Health care policy; Patient advocacy; Patient care; Quality care standards; Risk reduction; Severe asthma

\section{Key Summary Points}

Why carry out the study?

Patients with severe asthma use more health care resources, have more impaired well-being and quality of life, and die earlier than people in the general population

Poor health outcomes, overall negative effects on everyday life, and risk of death can increase when guidelines intended to improve medical care are not followed

The Improve Access to Better Care Task Force of the PRECISION Steering Committee was created to develop worldwide standards for managing severe asthma to help improve patient care

What was learned from the study?

A Quality Standard was developed as a guide to encourage key stakeholders to promote early identification and diagnosis of severe asthma, decrease delays to referral to specialists when cases are suspected, and result in effective management of severe asthma once diagnosed, with a particular focus on patient needs

Quality Statements, quality metrics, and local strategies are proposed to encourage effective use of these tactics to improve health care practices for patients with severe asthma

\section{INTRODUCTION}

Asthma is among the most commonly occurring non-communicable diseases, affecting approximately 339 million people worldwide [1], and is a substantial source of morbidity and mortality. Despite the availability of effective treatments, asthma ranks 16th among leading causes of years of life lived with disability [2] and 28th 
among leading causes of burden of disease [3]. Although severe asthma constitutes only about $10 \%$ of total asthma cases, these patients experience substantial burden of illness and account for up to $50 \%$ of asthma-related health care resources used [4]. Many patients are unable to access or afford needed care, contributing to the estimated 1000 people who die of asthma each day around the world [1]. Preventable asthma deaths continue to occur as a result of inappropriate management, including over-reliance on reliever medications, underutilization of preventive medications, and lack of understanding/awareness of available biomarkers to help personalize treatment decisions to optimize outcomes, particularly in patients with severe asthma [5-7].

Careful assessment of the suspected severe asthma patient is critical to determining the appropriate plan of care, as acute respiratory symptoms may be explained by alternative, or comorbid, conditions in some cases and require targeted intervention other than escalated asthma therapy [8]. When escalated asthma care is required, guideline-directed medical therapy (GDMT) is not widely implemented, and clinicians experience challenges staying abreast of evolving guidelines. For instance, Global Initiative for Asthma (GINA) recently published an update to its recommendations that, after 50 years as first-line care, short-acting $\beta$-agonist (SABA)-only is no longer recommended as initial therapy because of growing awareness of severe exacerbation risk [9]. GINA also recently began recommending increased caution when using add-on oral corticosteroids (OCS), based on increasing evidence of OCS-related adverse effects, coupled with the availability of OCSsparing biologic therapies $[9,10]$. Unfortunately, despite this update OCS use remains high [11]. While access to and especially the cost of newer therapies are significant barriers, limited awareness, understanding, or willingness to use biologic therapies introduced during the past several years may also underlie the delay in shifting away from the reliance on OCS.

Poor asthma management results in increased health care utilization and cost per patient [12], and lack of standardization in care is especially problematic for patients with severe asthma. Limited effectiveness of usual standards of care in preventing exacerbations that result in significant impairments in function and health-related quality of life (HRQOL) drive this increase in illness burden. While treatment for severe asthma may be up to five times more expensive than treatment for mild asthma in out-of-pocket costs [12], high-dosage OCS commonly used in these patients are associated with a high risk for short- and long-term adverse events, additional medical complications, and ultimately greater societal costs. Action is needed to address the unnecessary burden and premature mortality associated with severe asthma.

As outlined in the PRECISION-supported Charter to Improve Patient Care in Severe Asthma [13], patients with asthma should have access to early identification and diagnosis, timely referral for specialty care, appropriate treatment, and ongoing management and follow-up to improve outcomes, reduce cost and burden, and improve quality of life regardless of where they live (Fig. 1). That document served as a guide to the Improve Access to Better Care Task Force of the PRECISION Global Steering Committee (the Task Force) in the development of a global position on the standard of care for the management of severe asthma. This Global Quality Standard is intended to be a stimulus and guide for key stakeholders (e.g., governments, clinical leaders, policymakers, guideline writers, payers, advocacy groups) to help optimize implementation of care that allows for the early identification and diagnosis of severe asthma, timely referral to severe asthma specialists, and optimal treatment and ongoing management practices (Fig. 1).

\section{METHODS}

\section{Aims}

This design of the Global Quality Standard was informed by several considerations. First, Quality Standards should be practical and of direct use to clinicians, policymakers, commissioners, guideline writers, journalists, patient charity 


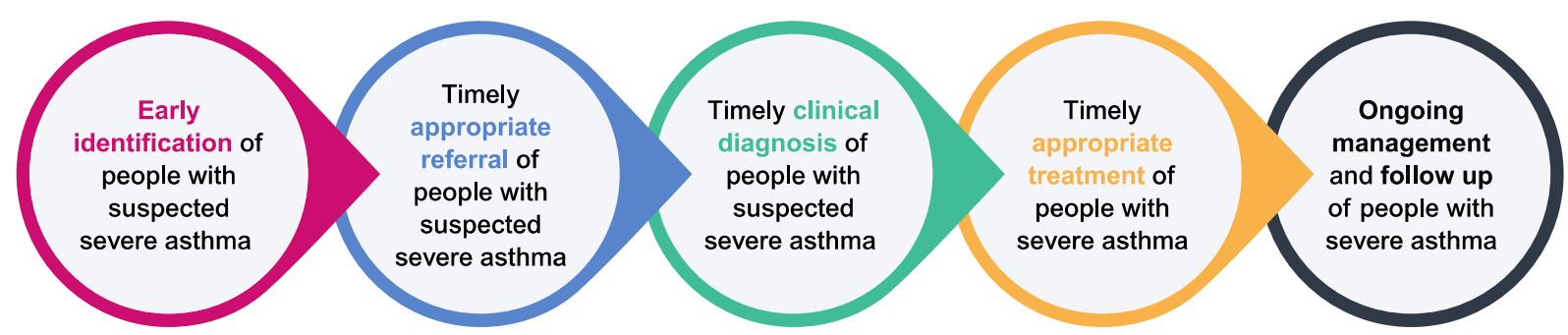

Fig. 1 The scope of the Global Quality Standard encompasses principles to address the unmet need and burden in severe asthma, with the intent to deliver improved care for patients with severe asthma

and advocacy groups, and asthma patients. Second, while aspirational, these standards should also be achievable by most well-equipped health care systems in developed countries. For optimal success, standards must be tailored to local requirements; therefore, national and local clinical groups worldwide must agree to and interpret standards for successful implementation. Accordingly, an implementation plan should be agreed upon by local leaders, and progress regarding process, outcomes, and uptake must be measured locally. Finally, this initiative is intended to allow for the comparison of standards achieved across health care systems, and to support the continual development and improvement of these systems to support improved patient care and outcomes. To that end, this document proposes both a list of potential metrics and areas in which localization is required to ensure optimal engagement and uptake. This article is based on Task Force consensus and does not contain any studies with human participants or animals performed by any of the authors.

\section{Standard Development}

The Task Force convened in January 2019 to support the development of a global position regarding the clinical management of patients with severe asthma for use by national and local clinical groups. During the course of six Task Force meetings and interim communications throughout 2019, consensus was gained for the Global Quality Standard for Severe Asthma summarized here. The four agreed upon Quality Statements, along with their supporting rationale and quality metrics to support local adaptation and implementation, are detailed below. This Quality Standard is based on the recently updated and empirically driven global clinical guidelines [9], as well as leading respiratory society recommendations for severe asthma care [14] and the PRECISION-supported Charter to Improve Patient Care in Severe Asthma [12], The severe asthma patient charter outlines patients' expectations and their rights to better care [13].

For the purposes of these standards, a diagnosis of severe asthma (or suspected severe asthma) is defined as asthma of sufficient severity to require the combination of highdosage inhaled corticosteroids and long-acting beta-agonists, and additional therapies or OCS to prevent the condition from becoming uncontrolled $[9,14]$.

\section{RESULTS}

The Task Force developed a consensus agreement on a Global Quality Standard for Severe Asthma, including four new Quality Statements, supporting rationale, metrics, and local adaptation guidance to support health care systems and providers in the administration of optimal care for patients with severe asthma. The intended audience includes national/international, regional, and local clinical groups, policymakers, commissioners, and guidelines writers. Figure 2 summarizes the core elements of the Global Quality Standard, and Table 1 summarizes the Quality Statements and their rationales. A detailed summary of each statement along with its supporting rationale, 
proposed metrics, and local adaptation opportunities follows.

\section{Element 1: Organization of Services}

\section{Quality Statement 1}

The health care system facilitates effective communication between providers and patients with diagnosed or suspected severe asthma to minimize a patient's daily symptoms, decrease the risk of asthma exacerbations, improve or reduce future loss of lung function, and reduce the risks of adverse events from OCS and other medications.

\section{Rationale}

Individuals with diagnosed or suspected severe asthma, and worsening symptoms or exacerbations, may visit hospitals, emergency departments, urgent care facilities, or other clinical settings. These medical events often have important implications for treatment planning. Effective decision-making requires awareness by every health care provider involved in a given patient's care of decisions made in other settings that may relate to the well-being and optimal care of the patient.

\section{Essential Criteria, Quality Metrics, and Local Adaptation Opportunities}

Criterion 1A: A clear referral network of care providers is required at local, regional, and national levels to connect general practitioners with asthma specialists and prevent delays in diagnosis and treatment.

\section{Potential Metrics}

(a) Number of total providers in the referral network.

(b) Percentage of clinicians within the network who specialize in, and are dedicated to, asthma care.

(c) Evidence of referral pathways and multidirectional information flow.

\section{Local Adaptation Opportunities}

(a) Provide structural updates to the network of severe asthma care providers (e.g., using a "hub and spoke model" wherein a main campus ["hub"] receives heavier resource investments proportionate to the number of asthma patients served and supplies the most intensive medical services, and is complemented by satellite campuses ["spokes"] that offer more limited services).

(b) Develop a dedicated list of asthma care providers and their specialist areas to be made available to all facilities and health care providers within the network.

(c) Ensure that services and health care providers are covered by the network (e.g., emergency department, community providers/clinics).

(d) Demonstrate evidence of effective communication between health care providers and facilities.

Criterion 1B: An infrastructure that enables real-time sharing of clinical data, including possible alerts when OCS and short-acting betaagonists (SABA) are prescribed, serious

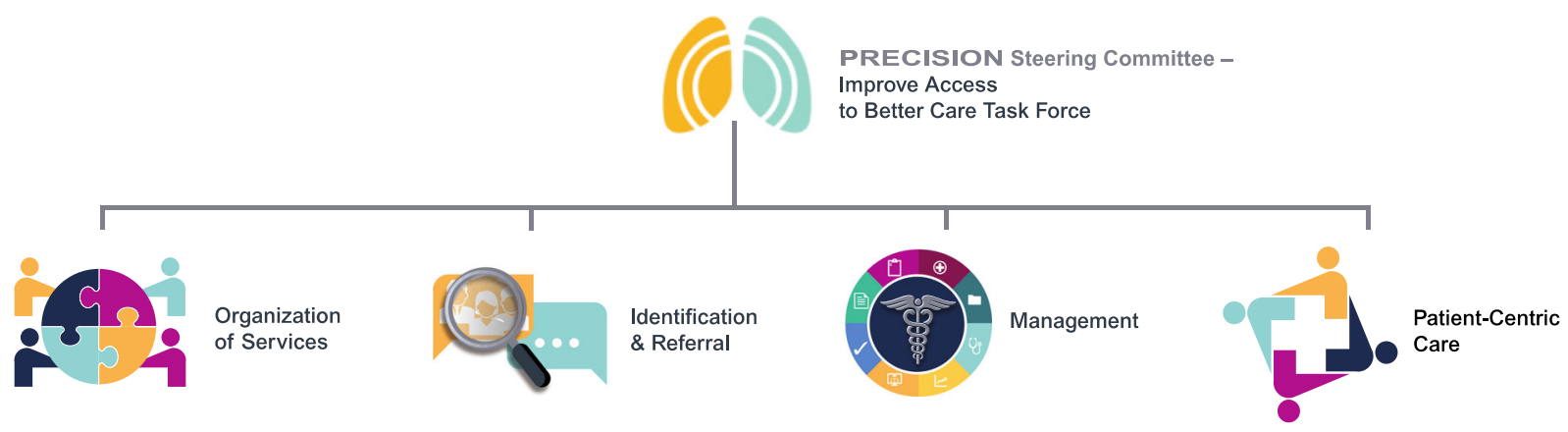

Fig. 2 The Global Quality Standard for improved management of severe asthma, as developed by the Improve Access to Better Care Task Force of the PRECISION Steering Committee, consists of four core elements. See Table 1 for more detail 
Table 1 Quality Statements and rationales for the Global Quality Standard for the treatment of severe asthma

\begin{tabular}{|c|c|c|}
\hline Category & Statement summary & Rationale \\
\hline $\begin{array}{c}\text { Organization } \\
\text { of services }\end{array}$ & $\begin{array}{l}\text { Health care systems should facilitate effective } \\
\text { communication between clinicians and patients to } \\
\text { reduce disease burden and risk of OCS adverse } \\
\text { events }\end{array}$ & $\begin{array}{l}\text { Patients with severe asthma seek care in various } \\
\text { settings, and communication is critical to effective } \\
\text { treatment planning and decision-making }\end{array}$ \\
\hline $\begin{array}{l}\text { Identification } \\
\text { and referral }\end{array}$ & $\begin{array}{l}\text { Early identification and specialty referral for patients } \\
\text { with severe or treatment-resistant asthma is } \\
\text { critical }\end{array}$ & $\begin{array}{l}\text { Severe asthma is often refractory to usual standards } \\
\text { of care and warrants specialty referral to facilitate } \\
\text { improved outcomes }\end{array}$ \\
\hline Management & $\begin{array}{l}\text { Phenotype assessment of patients with severe asthma } \\
\text { allows for precision-medicine-based care and the } \\
\text { possibility of improved outcomes }\end{array}$ & $\begin{array}{l}\text { Asthma is a heterogenous disease, and } \\
\text { understanding key biomarkers may optimize } \\
\text { decision-making and outcomes }\end{array}$ \\
\hline $\begin{array}{l}\text { Patient- } \\
\text { centric care }\end{array}$ & $\begin{array}{l}\text { Shared decision-making is key, and outcomes should } \\
\text { be monitored and documented via a personalized } \\
\text { asthma action plan }\end{array}$ & $\begin{array}{l}\text { Shared decision-making and ongoing assessment of } \\
\text { treatment benefit with a multidisciplinary team } \\
\text { are critical for optimizing clinical outcomes }\end{array}$ \\
\hline
\end{tabular}

OCS oral corticosteroids

exacerbation events are experienced by a patient, and/or following discharge from a hospital/emergency department.

\section{Potential Metrics}

(a) Number of clinicians using the same data infrastructure.

(b) Percentage of patients with asthma seen in the emergency department or admitted to hospital because of an asthma exacerbation, and the severity of their asthma.

(c) Percentage of providers with alerts for OCS prescribing (e.g., more than one prescription in the past year).

(d) Percentage of providers with alerts for SABA prescribing (e.g., more than three prescriptions in the past year).

\section{Local Adaptation Opportunities}

(a) Improve information technology solutions to allow clinicians across care settings ready access to clinical documentation relevant to managing a patient with severe asthma.

(b) Standardize data infrastructure across settings to improve access for general practitioners and asthma specialists to maximize consistency of advice and care provided to asthma patients.

(c) Standardize computerized alerts (content and process for delivery), and provide regular and standardized education to clinicians on what and how information will be provided.

Criterion 1C: A validated decision tool to help support informed decision-making by patients regarding their care, and accompanying health care provider support, are used.

\section{Potential Metrics}

(a) Percentage of patients using decision tool and availability of translated, adapted, and culturally appropriate versions for diverse populations.

(b) Number of downloads of the decision tool if available electronically.

(c) Annual survey of health care providers to evaluate frequency of dissemination of the decision tool to patients.

Local Adaptation Opportunities Standardization of content and format of a patient decision tool for use across clinical settings. 
Criterion 1D: When a suspected case of severe asthma is identified, the patient is evaluated by a multidisciplinary team (MDT) that can exclude or manage other pathology and provide treatment recommendations for "whole-patient" care.

\section{Potential Metrics}

(a) Number of facilities with an MDT available for assessment and management of patients with asthma.

(b) Percentage of facilities with an established protocol for utilizing an MDT for managing patients with asthma.

(c) Percentage of patients with a documented asthma action plan developed with an MDT.

Local Adaptation Opportunities An MDT may include specialties such as respiratory physicians, clinical nurse specialists, radiologists, pathologists, allergists, physiotherapists, clinical health psychologists, speech and language therapists, dietitians, and clinical pharmacists.

Criterion 1E: Health care providers involved in the care of patients with severe asthma receive updates on the diagnosis, treatment plan, and follow-up plan for these patients.

\section{Potential Metrics}

(a) Percentage of providers (general practitioners and specialists) in an MDT receiving an update following a significant change in a patient's status.

(b) Percentage of general practitioners receiving information related to formal patient diagnosis, relevant investigations undertaken, and treatment plan from the asthma specialist (for initial and subsequent visits, ideally within 2 weeks of the specialty clinic visit).

\section{Local Adaptation Opportunities}

(a) Standardize the format of formal diagnosis notification and/or alert. (b) Health care communities may wish to recommend suggested procedures for evaluating and treating patients with asthma (e.g., recommended tests or investigations, recommended treatment plan structure), and document when data (e.g., images or files) are provided to the patient.

\section{Element 2: Identification and Referral of Suspected Severe Asthma}

\section{Quality Statement 2}

People with difficult asthma who are unresponsive to optimal standard-of-care therapy are rapidly identified, reviewed, and referred to specialist care.

\section{Rationale}

People with diagnosed or suspected severe asthma may not respond to optimal treatment with (inhaled) standard-of-care therapies. The lack of response may be due to poor/suboptimal adherence or insufficient inhaler technique; however, some patients may continue to have severe uncontrolled asthma despite optimized medical management. Regardless of the reason, referral to specialist care is appropriate.

\section{Essential Criteria, Quality Metrics, and Local Adaptation Opportunities}

Criterion 2A: Patients with suspected severe asthma are referred to an asthma specialist for assessment (utilizing the established referral network; see criterion $1 \mathrm{~A}$, above).

\section{Quality Metrics}

(a) Percentage of individuals with uncontrolled asthma on locally agreed or GINA standard of care (stage $4+$ ).

(b) Percentage of patients with suspected severe asthma referred for diagnosis within a prespecified period of time (e.g., a number of months defined locally based on abilities and perspectives of the country/ region).

(c) Percentage of patients with suspected severe asthma in the general population who 
have not been referred to an asthma specialist.

(d) Percentage of patients with asthma in primary care for a prespecified period of time before referral to specialty asthma care (e.g., a number of weeks defined locally based on abilities and perspectives of the country/region).

(e) Percentage of patients with asthma receiving two or more courses of OCS without being managed in a specialty care service.

(f) Percentage of patients with asthma receiving three or more SABA in a 12-month period.

\section{Local Adaptation Opportunities}

(a) Generate a list of care settings that can refer to specialty care (e.g., general practice, secondary care, emergency department, community, urgent care), and implement a system to facilitate timely referral.

(b) Provide education about the role of patient self-referral for specialty asthma care.

(c) Establish local criteria for identification of suspected severe asthma.

(d) Establish local guidelines on markers of asthma control, including thresholds for OCS usage and SABA prescribing patterns that should trigger specialty referral.

(e) Establish local triggers for specialty referral (e.g., maximum threshold of OCS use, number of emergency department or urgent care visits within a defined time frame).

(f) Establish waiting time targets and thresholds for maximum time a patient with uncontrolled asthma should be managed in primary care.

(g) Availability of established guidelines to support clinicians with tapering strategies for corticosteroids, and exemptions.

Criterion 2B: Local leadership establishes a list of clinical assessment and investigation tools to disseminate to clinicians to improve identification of severe asthma, and implement when severe asthma is suspected.
Quality Metrics Percentage of patients with suspected severe asthma with documented completion of locally agreed upon clinical assessments/investigations within a prespecified period of time (e.g., a number of weeks defined locally based on abilities and perspectives of the country/region).

Local Adaptation Opportunities Generate a standardized list of clinical assessment and investigation tools and disseminate to clinicians/clinics for reference and implementation in practice.

Criterion 2C: Clinics/facilities have an asthma "champion" who is a peer leader/clinician trained in asthma assessment and management and receives ongoing support from the facility to serve in the role of change agent. The asthma champion is responsible to ensure asthma care standards are maintained, including availability and use of recommended tests and procedures, and continuing medical education (CME) is provided for all clinical staff treating patients with asthma.

\section{Quality Metrics}

(a) Percentage of health care providers with local access to an asthma champion in their facility/clinic.

(b) Percentage of clinical staff by practice type/ discipline receiving asthma-specific $\mathrm{CME}$ credits in a 12-month period.

\section{Local Adaptation Opportunities}

(a) Clarify scope of staff members and organizational involvement to support maintenance of standards and achievement of continuing education guidelines.

(b) Standardize CME content and format.

(c) Pursue accreditation for training curriculum to allow provision of formal CME credits to incentivize providers to complete training.

(d) Establish a timeline for CME refresher courses. 
Element 3: Management of Severe Asthma

\section{Quality Statement 3}

People with suspected severe asthma referred to specialists or other trained health care professionals will undergo a phenotype assessment to optimize asthma medications and promote precision-medicine-based care, thus increasing the chances of improving clinical outcomes.

\section{Rationale}

The underlying biologic drivers of severe asthma vary by patient, and are paramount to identification of severe asthma phenotypes. Understanding the biologic drivers of disease, and the key biomarkers that may better predict a patient's response to an individual therapy, is critical to optimize patient care and deliver superior clinical outcomes.

\section{Essential Criteria, Quality Metrics, and Local Adaptation Opportunities}

Criterion 3A: Individuals with severe asthma receive assessment for personalized phenotypebased treatment.

\section{Quality Metrics}

(a) Percentage of patients receiving biomarker tests.

(b) Percentage of health care providers with access to and who are utilizing tools required to properly assess phenotype, which should include, at a minimum:

i. Blood eosinophil (EOS) tests.

ii. Fractional exhaled nitric oxide (FeNO).

iii. Total immunoglobulin E (IgE).

iv. Spirometry.

(c) Percentage of patients receiving phenotype assessment, which should include, at a minimum, the tools/tests listed above.

\section{Local Adaptation Opportunities}

(a) Optimize the type of clinicians involved in assessment planning and interpretation.

(b) Standardize time to phenotype assessment.

(c) Centralize testing location.
Criterion 3B: Maintenance OCS should be considered an option of last resort, reserved only for patients who are ineligible for, or do not have access to (e.g., because of location, cost, or insurance coverage) biologic therapies.

\section{Quality Metrics}

(a) Percentage of patients receiving, or initiated on, maintenance OCS.

(b) Percentage of patients on maintenance OCS who have documented evidence of adherence to inhaled medications.

(c) Percentage of patients on maintenance OCS who are prescribed and take OCSsparing agents.

\section{Local Adaptation Opportunities}

(a) Establish local guidelines and tools to limit use of maintenance OCS.

(b) Establish local guidelines and tools to limit use of OCS in patients with newly identified asthma.

(c) Evaluate the total number of patients on maintenance OCS (e.g., via an annual report).

Criterion 3C: For patients currently taking OCS, the asthma action plan will include development of a corticosteroid-sparing strategy.

\section{Quality Metrics}

(a) Percentage of asthma action plans that include reference to OCS-sparing strategies.

(b) Percentage of patients with reduced use of OCS (within a 12-month cycle).

(c) Percentage of patients prescribed biologics for asthma maintenance care.

\section{Local Adaptation Opportunities}

(a) Develop local approaches and tools to support standard inclusion of OCS-sparing strategies as a standard in every asthma action plan.

(b) Develop and disseminate exemption criteria as guidance for network providers. 


\section{Element 4: Patient-Centric Care}

\section{Quality Statement 4}

Treatment decisions are made in partnership between the patient and clinician, and reflect the patient's expectations, priorities, and values. The impact of treatment is regularly reviewed and tracked in a personalized asthma action plan.

\section{Rationale}

The goals of management of severe asthma are to reduce the risk of severe exacerbations and improve daily symptom control. Clinicians are reminded of the need to treat the person, not the diagnosis, with consideration of comorbid conditions and psychosocial factors. With the increased number of "informed patients," shared decision-making is more likely to lead to better clinical outcomes.

As severe asthma is a chronic, variable condition, periodic reviews are reasonable to assess whether the condition has changed, to consider if alternative intervention is appropriate, and to offer ongoing education, training, and support. Like most chronic conditions, asthma is associated with various physical and psychosocial sequelae and comorbidities. Tracking and documenting comprehensive changes as part of a dedicated asthma action plan ensures that all providers involved in the care of a patient with severe asthma can offer the most informed care.

\section{Essential Criteria, Quality Metrics, and Local Adaptation Opportunities}

Criterion 4A: Patients with severe asthma receive relevant information and education sufficient to support participation in shared decisionmaking.

\section{Quality Metrics}

(a) Percentage of severe asthma patients offered personalized information related to their types of severe asthma at the time of their formal diagnoses, and at follow-up visits.

(b) Evaluate perceived value (to patients) of providing personalized information about asthma during clinic visits. (c) Percentage of clinical staff with up-to-date education and training to support shared decision-making.

\section{Local Adaptation Opportunities}

(a) Standardize the format and provision of patient information. For example:

i. If education is to be provided in a formal educational program/lecture, standardize content, recruitment, and staff delivering materials to patients and families.

ii. If education is provided during informal personalized clinical conversations with a health care provider, standardize the information to be provided/ reviewed.

iii. If handouts or leaflets are used, ensure the printed information is updated with the most recent best practice data and is appropriate for the population served (e.g., appropriate reading level, language, alternative formats for persons with unique learning challenges or disabilities).

iv. Standardize the scope of information included in patient education materials according to consensus among experts in severe asthma networks, while ensuring adapted, translated, and culturally appropriate versions are available for diverse populations in a format personalized for each patient/family.

Criterion 4B: Patients with severe asthma receive a periodic review of their condition and the impact of management approaches on outcomes.

\section{Quality Metrics}

(a) Percentage of patients receiving periodic follow-up for their asthma.

(b) Percentage of patients attending asthma review appointments with an asthma specialist. 
(c) Percentage of asthma reviews that include an assessment of psychosocial issues and function, in addition to comorbidities.

(d) Additional agreed upon parameters for review (e.g., level of symptom control, ED visits, hospital admissions, lung function, inhaler technique review, frequency of use of preventer and reliever medications) that are documented in the patient medical record.

\section{Local Adaptation Opportunities}

(a) Establish protocols to standardize the regularity of periodic review with patients.

(b) Establish protocols to standardize an explicit process of managing patients who fail to attend specialist asthma review visits.

(c) Establish local protocols for inclusion of psychosocial issues in periodic review with patients.

Criterion 4C: Criteria for a periodic review will be established by local clinical leadership, and published for easy access and use by local clinical providers (i.e., "A periodic review should include..." with criteria to be explicitly documented by the local leadership).

Quality Metrics Percentage of locally agreed review criteria conducted/completed in the specified time frame.

Local Adaptation Opportunities Criteria constituting a periodic review may include:

i. Measurement of asthma and/or symptom control.

ii. Inhaler technique assessment and review.

iii. Recording of exacerbation treatment and frequency of need for steroids.

iv. Number of emergency department visits.

v. Number of hospital admissions.

vi. Treatment adherence.

vii. Avoidance of adverse effects.

viii. Prompts for prescription refills.

ix. Prompts for vaccinations.

$\mathrm{x}$. FeNO, spirometry, and blood EOS tests.

xi. Tobacco use/smoking behavior.

xii. Exercise behavior.
Criterion 4D: Patients have a dedicated asthma action plan in which changes and decisions related to their asthma care are integrated and well documented.

Quality Metrics Percentage of patients with a personalized asthma action plan reflective of care needs, including personalized contact with health care professionals for advice, counsel, and shared decision-making opportunities.

\section{Local Adaptation Opportunities}

(a) Establish criteria to standardize asthma action plan format and content.

(b) Establish criteria for updating the asthma action plan.

Criterion 4E: Patient records are owned by the patient and available to health care professionals involved in the patient's care.

\section{Quality Metrics}

(a) Percentage of organizations involved in care of patients with severe asthma that are able to access patient medical records, including the asthma action plan.

(b) Percentage of patients with access to, or in possession of, their own medical records.

(c) Percentage of patients sharing their medical record data with members of the MDT.

Local Adaptation Opportunities Expand the scope of accessibility of patient data sharing.

i. Health care professionals in the MDT should be able to easily access relevant medical information for their patient with severe asthma. Reducing barriers to access allows for greater personalization of the health care experience. However, access history is auditable to ensure protection of patient privacy.

ii. Patients should have easy access to their medical records, preferably available in an easily understandable format. Patients should be given the opportunity to discuss data contained in the medical record with a professional who can assist them in interpreting and understanding information, 
particularly in cases in which learning, reading, or cognitive capacity may be limited.

\section{DISCUSSION}

Severe asthma is a substantial source of morbidity, mortality, and disease burden across the world. Despite an extensive evidence base and other published clinical guidelines focused on the management of severe asthma [9], patients may not receive GDMT and therapeutic goals are not reached. In severe asthma, current treatment practices include a continued overreliance on OCS, and underuse of therapies that can prevent, or substantially reduce, the frequency of exacerbations and associated cost and reduction in HRQOL. Moreover, an accurate diagnosis followed by precision-medicine-based assessment practices that could support treatment personalization and optimization.

Health care providers need education to better understand severe asthma, and clinical guidelines on the assessment and treatment of the condition. In addition, improving the treatment landscape requires systems-level changes to enhance awareness of (and access to) specialty referral options and strategies to reduce communication barriers between providers/clinical teams within health care systems. These may include increased access to, and use of, new technologies such as telemonitoring and digital reporting of treatment use patterns from electronic inhalers, which may help delay progression to severe asthma $[15,16]$. Until such improvements are made, patients will continue to experience more frequent asthma exacerbations, emergency care visits, hospital admissions, and the sequelae of psychological, social, and occupational impairments commonly associated with a poorly controlled, chronic illness.

The PRECISION Steering Committee convened to address the urgent need for review of the current asthma care landscape, and support improvements in standardization of care and access to GDMT for severe asthma. The PRECISION-supported Charter to Improve Patient Care in Severe Asthma [13], which outlined six core principles to educate and mobilize key stakeholders, was an important first step to improve awareness of and access to quality care for severe asthma.

The Global Quality Standard outlined in this report, which is based on the vast body of published evidence available for asthma care and published clinical guidelines, was the key product produced by the Task Force endeavors. The proposed standard emphasizes core elements (see Table 1) that are reflective of the patients' expectations, priorities, and values. It is aimed at optimizing clinical care and outcomes for all patients with severe asthma and reducing associated cost and burden (at the level of the individual, society, and health care system).

Governments, payer policymakers, guideline writers, clinicians, patients, and caregivers must unite and mobilize to achieve meaningful improvements in severe asthma care. The continuum of care detailed herein is relevant to local, regional, national, and global health care systems and partners, and successful implementation will rely upon thoughtful adaptation based on the local needs, strengths, and weaknesses of a clinical system. These standards can be adapted to meet the needs of any local health care system regardless of available resources, communication/medical record access, referral network organization, or knowledge and use of GDMT. For optimal success, standards must be tailored to local requirements, and an implementation plan should be agreed upon by local leaders. Moreover, progress (e.g., process implementation, outcomes, and uptake) must be determined locally, with the goal to compare between health care systems to support continual development and improvement necessary to optimize patient care and outcomes. We urge policymakers, health care providers, and patient advocacy groups to employ the Quality Standard set forth in this document, in addition to the core principles outlined in the complementary PRECISION-supported Charter to Improve Patient Care in Severe Asthma [13]. These resources will help achieve consensus on the definition of quality care in severe asthma, promote patient-centric care, identify gaps in 
care and areas for improvement, and systematically implement quality care improvement measures to improve outcomes and reduce the burden of illness in patients with severe asthma.

\section{CONCLUSIONS}

This Quality Standard for severe asthma detailed by the Improve Access to Better Care Task Force of the PRECISION Steering Committee is well supported by published clinical guidelines, and serves as a complement to the 2018 PRECISION-supported Charter to Improve Patient Care in Severe Asthma. Key stakeholders (e.g., governments, policymakers, clinicians, payers, patient advocacy groups) are encouraged to use this Quality Standard to guide important collaborative efforts needed to standardize clinical care and optimize outcomes for patients with severe asthma around the world.

\section{ACKNOWLEDGEMENTS}

The authors thank the PRECISION Global Steering Committee and the PRECISION Improve Access to Better Care Task Force. The PRECISION program is an AstraZeneca-sponsored global initiative to help people living with severe asthma have access to appropriate treatment and care. PRECISION Improve Access to Better Care Task Force members: Hannah Saul ${ }^{1}$, Tonya Winders ${ }^{2}$, Giorgio Walter Canonica ${ }^{3}$, Pascal Chanez ${ }^{4}$, Janwillem Kocks ${ }^{5}$, John Haughney ${ }^{6}$, Steve Holmes ${ }^{7}$, Adel Mansur ${ }^{8}$, Andrew Menzies-Gow ${ }^{9}$, Yuji Tohda ${ }^{10}$, Cynthia Hallensleben $^{11}$, Grainne d'Ancona ${ }^{12}$, Sam Prigmore ${ }^{13}$, Esther Metting ${ }^{14}$. ${ }^{1}$ AstraZeneca, Cambridge, UK; ${ }^{2}$ Allergy \& Asthma Network, Vienna, VA, USA; ${ }^{3}$ Humanitas University, Razzano, Italy; ${ }^{4}$ Aix Marseille Université, Clinique des bronches, allergie et sommeil/ APHM, Marseille C2VN Center INSERM INRA UMR1062, Marseille, France; ${ }^{5}$ University of Groningen, Groningen, Netherlands; ${ }^{6}$ Glasgow Clinical Research Facility, Queen Elizabeth University Hospital, Institute of Neurological Sciences, Glasgow, UK; ${ }^{7}$ Park Medical Practice, Shepton Mallet, UK; ${ }^{8}$ Heartlands Hospital, Birmingham,
UK; ${ }^{9}$ Royal Brompton and Harefield NHS Foundation Trust, London, UK; ${ }^{10}$ Kindai University, Osaka, Japan; ${ }^{11}$ Leiden University Medical Center, Leiden, Netherlands; ${ }^{12}$ Guys and St. Thomas' NHS Foundation Trust, London, UK; ${ }^{13}$ St. George's Hospital NHS Foundation Trust, London, UK; ${ }^{14}$ Groningen Research Institute, Groningen, Netherlands.

Funding. AstraZeneca (Gaithersburg, MD, USA) funded this study and the journal's Rapid Service Fee and Open Access publication. All authors had full access to the content and take complete responsibility for its integrity and accuracy.

Medical Writing, Editorial, and Other Assistance. Editorial and writing support, including preparation of the draft manuscript under the direction and guidance of the authors, incorporation of author feedback, and manuscript submission, was provided by Teresa E. Fecteau, PhD (CiTRUS Health Group, Cleveland, $\mathrm{OH}$, USA), and Michael A. Nissen, ELS (AstraZeneca, Gaithersburg, MD, USA). This support was funded by AstraZeneca.

Authorship. All named authors meet the International Committee of Medical Journal Editors criteria for authorship for this article, take responsibility for the integrity of the work as a whole, and have given their approval for this version to be published.

Disclosures. John Haughney has consultancy agreements with AstraZeneca for the PRECISION program, and has received consulting fees from AstraZeneca for past work regarding management of chronic obstructive pulmonary disease in the UK. Tonya A. Winders has consultancy agreements with AstraZeneca for the PRECISION program. Steve Holmes has received funding from AstraZeneca, Beximco, Boehringer Ingelheim, Chiesi, GlaxoSmithKline, Johnson and Johnson, Mylan, Napp, Novartis, Nutricia, Orion, Pfizer, Sandoz, Teva, and Trudell Medical International. Pascal Chanez has consultancy agreements with AstraZeneca for the PRECISION program, and has received research and consulting funding from 
AstraZeneca, Amirall, Boehringer Ingelheim, Centocor, GlaxoSmithKline, Merck Sharp \& Dohme, Novartis, Teva, Chiesi, and Schering Plough. Hannah Saul is employed by AstraZeneca as Global Director of Advocacy and Policy, Respiratory and INA. Andrew Menzies-Gow has consultancy agreements with AstraZeneca, Sanofi and Vectura; was an advisory board member for AstraZeneca, Sanofi, GlaxoSmithKline, Novartis, and Teva; received speaker fees from AstraZeneca, Sanofi, Novartis and Teva; has received clinical funding from AstraZeneca; and has attended international conferences sponsored by Teva.

Compliance with Ethics Guidelines. This article is based on Task Force consensus and does not contain any studies with human participants or animals performed by any of the authors.

Data Availability. Data sharing is not applicable to this article as no datasets were generated or analyzed during the current study.

Open Access. This article is licensed under a Creative Commons Attribution-NonCommercial 4.0 International License, which permits any non-commercial use, sharing, adaptation, distribution and reproduction in any medium or format, as long as you give appropriate credit to the original author(s) and the source, provide a link to the Creative Commons licence, and indicate if changes were made. The images or other third party material in this article are included in the article's Creative Commons licence, unless indicated otherwise in a credit line to the material. If material is not included in the article's Creative Commons licence and your intended use is not permitted by statutory regulation or exceeds the permitted use, you will need to obtain permission directly from the copyright holder. To view a copy of this licence, visit http:// creativecommons.org/licenses/by-nc/4.0/.

\section{REFERENCES}

1. Global Asthma Network. The global asthma report 2018. Auckland, New Zealand: Global Asthma Network, 2018. https://www.globalasthmareport. org/Global\%2520Asthma\%2520Report\%25202018. pdf. Accessed Mar 5, 2020.

2. Vos T, Abajobir AA, Abate KH, et al. GBD 2016 Disease and Injury Incidence and Prevalence Collaborators. Global, regional, and national incidence, prevalence, and years lived with disability for 328 diseases and injuries for 195 countries, 1990-2016: a systematic analysis for the Global Burden of Disease Study 2016. Lancet. 2017;390: 1211-59.

3. Hay SI, Abajobir AA, Abate KH, et al. GBD 2016 DALYs and HALE Collaborators. Global, regional, and national disability-adjusted life-years (DALYs) for 333 diseases and injuries and healthy life expectancy (HALE) for 195 countries and territories, 1990-2016: a systematic analysis for the Global Burden of Disease Study 2016. Lancet. 2017;390:1260-344.

4. World Allergy Organization. The management of severe asthma: economic analysis of the cost of treatments for severe asthma. 2005. https://www. worldallergy.org/educational_programs/world_ allergy_forum/anaheim2005/blaiss.php. Accessed Mar 5, 2020.

5. D'Amato G, Vitale C, Molina A, et al. Asthma-related deaths. Multidiscip Respir Med. 2016;11:37.

6. Price D, Bjermer L, Bergin DA, Martinez R. Asthma referrals: a key component of asthma management that needs to be addressed. J Asthma Allergy. 2017;10:209-23.

7. Royal College of Physicians. Why asthma still kills. The National Review of Asthma Deaths (NRAD). Confidential Enquiry report. London: Royal College of Physicians; May 2014. https://www.rcplondon. ac.uk/projects/outputs/why-asthma-still-kills. Accessed Mar 5, 2020.

8. Heaney LG, Robinson DS. Severe asthma treatment: need for characterising patients. Lancet. 2005;365(9463):974-6.

9. Global Initiative for Asthma. Global strategy for asthma management and prevention. Updated 2020. www.ginasthma.org. Accessed July 8, 2020.

10. Lefebvre P, Duh MS, Lafeuille MH, et al. Acute and chronic systemic corticosteroid-related complications in patients with severe asthma. J Allergy Clin Immunol. 2015;136(6):1488-95. https://doi.org/10. 1016/j.jaci.2015.07.046. 
11. Tran TN, King E, Sarkar R, et al. Oral corticosteroid prescription patterns for asthma in France, Germany, Italy, and the UK. Eur Respir J. 2020;55(6):1902363.

12. Bhandori K, Doyle-Waters MM, Marra C, et al. Economic burden of asthma: a systematic review. BMC Pulm Med. 2009;9:24.

13. Menzies-Gow A, Canonica GW, Winders TA, Correia Sousa J, Upham JW, Fink-Wagner AH. A charter to improve patient care in severe asthma. Adv Ther. 2018;35:1485-96.

14. Chung KF, Wenzel SE, Brozek JL, et al. International ERS/ATS guidelines on definition, evaluation and treatment of severe asthma. Eur Respir J. 2014;43: 343-73.

15. Sulaiman I, Greene G, MacHale E, et al. A randomised clinical trial of feedback on inhaler adherence and technique in patients with severe uncontrolled asthma. Eur Respir J. 2018;51(1): 1701126.

16. Merchant RK, Inamdar R, Quade RC. Effectiveness of population health management using the propeller health asthma platform: a randomized clinical trial. J Allergy Clin Immunol Pract. 2016;4(3): 455-63. 